\title{
Characteristics and outcomes of hemodialysis patients with COVID-19: a retrospective single center study
}

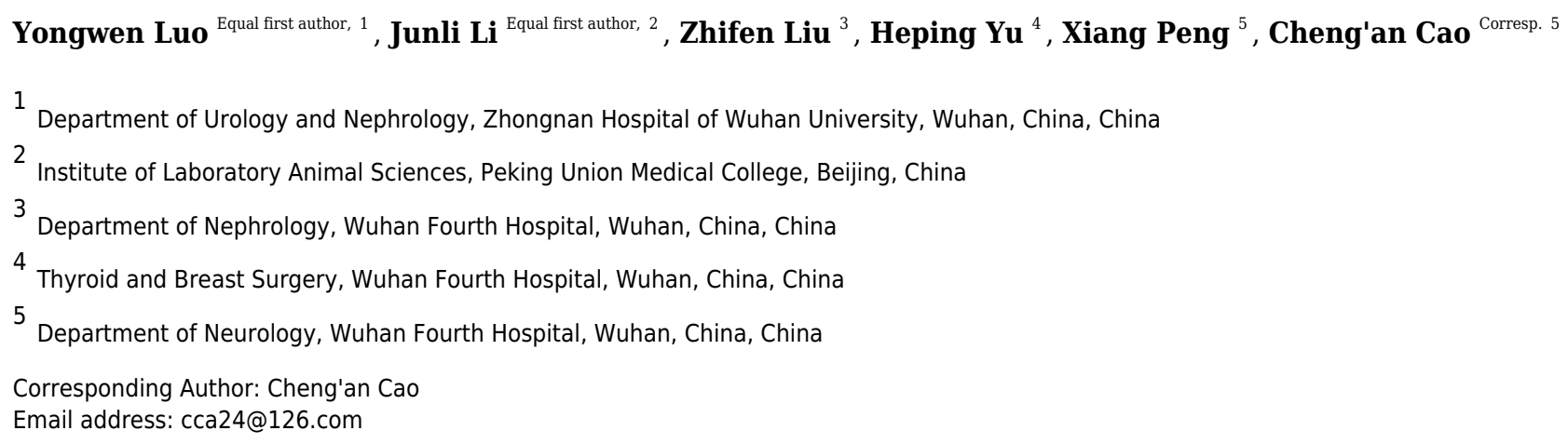

Background: The coronavirus 19 (COVID-19) pandemic has heightened the threat to the health and lives of patients with comorbid diseases. Infection by COVID-19 is especially detrimental to patients on hemodialysis. In this study, we evaluated the clinical characteristics, laboratory findings, treatments, and prognoses of hemodialysis patients with COVID-19.

Methods: 16 hemodialysis patients with COVID-19 were recruited from Wuhan Fourth Hospital from February $5^{\text {th }}$ to March $20^{\text {th }}, 2020$ for a retrospective, single-center study. 62 non-dialysis patients with COVID-19 were the control group. We collected data on the clinical characteristics, laboratory findings, treatments, and clinical outcomes of patients affected by the virus.

Results: Hemodialysis patients with COVID-19 had a lower incidence of fever $(P=0.001)$ and relatively higher incidence of pre-admission comorbidities and shortness of breath than non-dialysis patients with COVID-19 ( $75 \%$ vs $61 \%, P=0.46750 \%$ vs $33.87 \%, P=0.248$ ). Hemodialysis patients had lower levels of hemoglobin $(P<0.001)$, white blood cell counts $(P=0.015)$, neutrophils $(P=0.016)$, AST $(P=0.037)$, ALT $(P<0.001)$ and procalcitonin $(P<0.001)$, and higher levels of D-dimer $(P<0.001)$ and thrombin time $(P<0.001)$. Hemodialysis patients had a higher incidence of pulmonary effusion, cord-like highdensity shadows, pleural thickening, and atelectasis $(P<0.05)$. Hemodialysis patients also had relatively higher rates of mortality and prolonged hospital stays compared with the control group.

Conclusions: Hemodialysis patients typically present with multiple comorbidities and are considered to be a high-risk group for COVID-19 infections. Hemodialysis patients with COVID-19 may have prolonged hospital stays and unfavorable prognoses and should be closely monitored. 


\section{Characteristics and outcomes of hemodialysis patients with COVID-19: a}

2

3

4

5

\section{retrospective single center study}

Yongwen Luo, MD ${ }^{1 \#}$; Junli Li, PhD ${ }^{2 \# ; ~ Z h i f e n ~ L i u, ~ M D ³ ~}{ }^{3}$ Heping Yu, MD ${ }^{4}$; Xiang Peng, MD; Cheng'an Cao, $\mathrm{MD}^{5^{*}}$

${ }^{1}$ Department of Urology, Zhongnan Hospital of Wuhan University, Wuhan, China.

${ }^{2}$ Institute of Laboratory Animal Sciences, Chinese Academy of Medical Sciences (CAMS), Comparative Medicine Center, Peking Union Medical College (PUMC), Beijing, China.

${ }^{3}$ Department of Nephrology, Wuhan Fourth Hospital, Puai Hospital, Tongji Medical College, Huazhong University of Science and Technology, Wuhan, China.

${ }^{4}$ Department of Thyroid and Breast Surgery, Wuhan Fourth Hospital, Puai Hospital, Tongji Medical College, Huazhong University of Science and Technology, Wuhan, China.

${ }^{5}$ Department of Neurology, Wuhan Fourth Hospital, Puai Hospital, Tongji Medical College, Huazhong University of Science and Technology, Wuhan, China.

\# These authors contributed equally to this work.

*Corresponding author: Dr. Cao, Email: cca24@163.com, Address: No. 473 Hanzheng Street, Qiaokou District, Wuhan, Hubei, China. 
18 Abstract

Background: The coronavirus 19 (COVID-19) pandemic has heightened the threat to the health and lives of patients with comorbid diseases. Infection by COVID-19 is especially detrimental to patients on hemodialysis. In this study, we evaluated the clinical characteristics, laboratory findings, treatments, and prognoses of hemodialysis patients with COVID-19.

Methods: 16 hemodialysis patients with COVID-19 were recruited from Wuhan Fourth Hospital from February $5^{\text {th }}$ to March $20^{\text {th }}, 2020$ for a retrospective, single-center study. 62 non-dialysis patients with COVID-19 were the control group. We collected data on the clinical characteristics, laboratory findings, treatments, and clinical outcomes of patients affected by the virus.

Results: Hemodialysis patients with COVID-19 had a lower incidence of fever $(P=0.001)$ and relatively higher incidence of pre-admission comorbidities and shortness of breath than nondialysis patients with COVID-19 (75\% vs $61 \%, P=0.46750 \%$ vs $33.87 \%, P=0.248$ ). Hemodialysis patients had lower levels of hemoglobin $(P<0.001)$, white blood cell counts $(P=0.015)$, neutrophils $(P=0.016)$, AST $(P=0.037)$, ALT $(P<0.001)$ and procalcitonin $(P<0.001)$, and higher levels of D-dimer $(P<0.001)$ and thrombin time $(P<0.001)$. Hemodialysis patients had a higher incidence of pulmonary effusion, cord-like high-density shadows, pleural thickening, and atelectasis $(P<0.05)$. Hemodialysis patients also had relatively higher rates of mortality and prolonged hospital stays compared with the control group.

Conclusions: Hemodialysis patients typically present with multiple comorbidities and are considered to be a high-risk group for COVID-19 infections. Hemodialysis patients with COVID19 may have prolonged hospital stays and unfavorable prognoses and should be closely monitored. 


\section{Introduction}

41 The coronavirus 2019 (COVID-19) pandemic caused by severe acute respiratory syndrome 42 coronavirus 2 (SARS-CoV-2) has created a major public health issue (CDC COVID-19 Response 43 Team, 2020). There have been 24,257,989 confirmed cases and 827,246 deaths reported across 44209 countries or regions since August 28 $8^{\text {th }}, 2020$ (WHO, 2020). Previous studies have shown that 45 older individuals, particularly those with chronic comorbidities, are generally more susceptible to 46 infections by COVID-19 (Chen et al., 2020; Wu \& McGoogan, 2020). 41.3\% of COVID-19 cases

47 occurred due to hospital-related transmissions (Wang et al., 2020a). Hemodialysis patients require

48 hospital stays for treatments multiple times per week and are already immunocompromised due to

49 uremia. Many of these patients are also elderly and have comorbidities including coronary disease,

50 hypertension, diabetes, and lung disease that are associated with unfavorable outcomes in patients

51 with COVID-19 (Zhou et al., 2020). Hemodialysis patients are therefore more highly susceptible

52 to infection by COVID-19 than the general population (Wang et al., 2020b; Xiong et al., 2020).

53 There is a particular interest in the clinical features and outcomes of hemodialysis patients infected

54 with COVID-19. Early research has found that the clinical symptoms of COVID-19, including 55 cough and fever, were less common in patients on hemodialysis but that hemodialysis patients 56 with COVID-19 had a higher risk of death than the general population (Tortonese et al., 2020; Wu

57 et al., 2020; Yang et al., 2020). Therefore, we evaluated the clinical characteristics, laboratory 58 findings, radiological characteristics, treatments, and outcomes of 16 hemodialysis patients with confirmed COVID-19 infections in order to provide an insight into the clinical assessment and 
60

\section{Patients and Methods}

\section{Study design and participants}

\section{Data collection}

management of hemodialysis patients with COVID-19.

We recruited hemodialysis patients with confirmed COVID-19 infections from Wuhan Fourth Hospital, Tongji Medical College, Huazhong University of Science and Technology, Wuhan, China from February $5^{\text {th }}$ to March $20^{\text {th }}, 2020$ for our retrospective, single-center study. Wuhan Fourth Hospital was designated as the treatment center for hemodialysis patients with COVID-19 during the outbreak and patients with confirmed or suspected COVID-19 infections were centrally admitted. All hemodialysis patients enrolled in this study had confirmed COVID-19 infections diagnosed according to the interim guidance by the WHO (CDC, 2020). We compared the results from hemodialysis patients with a control group of non-dialysis COVID-19 patients at the same hospital. We adjusted for age and sex and matched non-hemodialysis COVID-19 patients for sex and age by randomly selecting each hemodialysis patient with COVID-19 according to previously reported methods (Garcia et al., 2012; Lovshin et al., 2018). Our study was approved by the Institutional Review Board (IRB) at Wuhan Fourth Hospital (IRB approval number: KY 2020032-01). Informed consent was waived as part of the public health outbreak investigation.

Two doctors in our team gathered clinical information from electronic medical records. Patient information included epidemiology, demographics, medical history, laboratory findings, comorbidities, treatment regimens (antiviral, antibiotic, corticosteroid therapies, immune glucocorticoid therapy, and respiratory support), length of hospital stay, and clinical outcomes. 
81 Data that was missing from the medical records were obtained through direct communication with

82 attending doctors or the patients themselves.

83

\section{Definitions}

Discharge criteria for patients were defined as: a normal body temperature for more than three days, significant improvement in respiratory symptoms, improved chest $\mathrm{CT}$ imaging indicating reduced inflammation, and two consecutive negative nucleic acid tests throat swabs with at least a one-day interval between tests. A comprehensive evaluation was made by an expert team to determine whether the patient could be discharged.

\section{Statistical analysis}

Categorical variables were expressed as numerical values (\%) and continuous variables were presented as median with interquartile ranges (IQR) in the descriptive statistics. We compared the means for discrete variables using independent Student's t tests when the data were normally distributed. The Mann-Whitney test was used for data not normally distributed. Proportions for categorical variables were compared using the $\chi 2$ test, although the Fisher's exact test was used when the sample sizes were small. All statistical analyses were performed using SPSS software (version 21.0), and $P<0.05$ was considered statistically significant.

\section{Results}

\section{Clinical characteristics}

16 hemodialysis patients with COVID-19 were included in our study. The clinical characteristics of the 16 patients are shown in Table 1. The median patient age was 61 years (interquartile range 
102 103 104 105 106 107 108 109 110 111 112 113 114 115 116 117 118 119 120 121 122

54-78 years), and 8 of the 16 patients were female. None of the patients had exposure to the Huanan seafood market that appeared to be the epicenter of the COVID-19 outbreak. All hemodialysis patients studied had a history of being in contact with the epidemic area and of being in contact with patients who had a fever. Twelve cases $(75 \%)$ had comorbidities including coronary disease, hypertension, and diabetes. The most common initial symptoms at admission were: cough (75\%), shortness of breath (50\%), fatigue (50\%), and fever $(43.75 \%)$. The median incubation period was 9 days (interquartile range 4.3-12 days). Hemodialysis patients presented with a lower incidence of fever compared with non-dialysis patients $(P=0.001)$. Hemodialysis patients also had higher incidence of preadmission comorbidities and shortness of breath, although the difference was not significant.

\section{Radiological and laboratory findings}

16 patients $(100 \%)$ presented with abnormal chest scans at admission. The scans revealed typical signs of infection for COVID-19 in hemodialysis patients (Figure 1), such as bilateral patchy shadowing (100\%) and ground-glass opacities (62.5\%) (Table S1). Hemodialysis patients presented with a higher incidence of pulmonary effusion $(P<0.001)$, cord-like high-density shadows $(P<0.001)$, pleural thickening $(P=0.0046)$, atelectasis $(P=0.0046)$ and consolidation of lung tissues $(P=0.053)$ when compared with non-dialysis patients (Table 2). $15(93.75 \%)$ hemodialysis patients had anemia at admission and $14(92.86 \%)$ had coagulopathy with elevated D-dimer, while all hemodialysis patients presented with hypoproteinemia (100\%) and lymphopenia (100\%). Infection-related biomarkers, including procalcitonin and C-reactive protein, were also abnormal in almost all patients (Table S1). Hemodialysis patients had lower 
123 levels of hemoglobin $(P<0.001)$, white blood cell counts $(P=0.015)$, neutrophils $(P=0.016)$,

$124 \operatorname{AST}(P=0.037), \operatorname{ALT}(P<0.001)$ and procalcitonin $(P<0.001)$, and higher levels of D-dimer $(P$

$125<0.001)$ and thrombin time $(P<0.001)$ when compared with non-dialysis patients (Table 2).

126 Treatment and outcomes

All patients received treatment according to the Chinese Diagnosis and Treatment Protocol

for COVID-19 (6 $6^{\text {th }}$ version) (CDC, 2020). 16 patients received oxygen therapy in while in isolation

and 14 patients received antiviral treatment, including Arbidol (0.2 g three times daily, orally),

Lopinavir and Ritonavir tablets (0.5 mg twice daily, orally), Oseltamivir (75 mg twice daily, orally)

or Ribavirin (500 mg once per day, intravenously). Traditional Chinese medicines, such as

Lianhuaqingwen capsules and Toujiequwen Keli, were also given. 14 patients (87.5\%) were

treated with antibiotics, including Amoxicillin clavulanate potassium, Levofloxacin,

Moxifloxacin, Cephalosporin, Ceftriaxone, Cefoxitin, or Cefoperazone sulbactam. Nine patients

$(56.25 \%)$ received a single antibiotic treatment and five patients $(31.25 \%)$ received a combination

of treatments. Two patients $(12.5 \%)$ also received corticosteroid treatment, and one patient

(6.25\%) received intravenous immunoglobulin therapy (Table S2). There was no difference

between hemodialysis and non-dialysis patients in terms of treatment modality (Table S3).

Hemodialysis was performed with the usual frequency in an isolation room designated for COVID-

19 patients. We analyzed the clinical outcomes of the study participants at the end of the follow-

up period on April 5 $5^{\text {th }}, 2020$. The median period of hospitalization was 21 days (interquartile range,

15.5-30.25 days). 13 patients recovered and were discharged from the hospital and 3 patients died,

resulting in a mortality rate of $18.75 \%$. Hemodialysis patients showed higher mortality and a 
144 prolonged hospital stay, although the results were not significantly different $(P=0.427$ and $P=$ 1450.077 , respectively).

\section{Discussion}

148 The effect of COVID-19 on hemodialysis patients is of great interest to clinicians. Our retrospective, single-center study details the clinical characteristics and outcomes of hemodialysis patients infected with SARS-CoV-2.

We found that hemodialysis patients presented with similar clinical symptoms as patients with COVID-19 from the general population. Symptoms included: fever, cough, sore throat, shortness of breath, myalgia, headache, fatigue, and diarrhea (Wu \& McGoogan, 2020; Xiong et al., 2020). The incubation period lasted approximately 1-14 days in both groups. A previous study reported that $18.7 \%$ of non-dialysis patients had shortness of breath (Xiong et al., 2020), but 50\% of hemodialysis patients experienced shortness of breath, which may be attributed to several factors.

157 Firstly, half of the hemodialysis patients (50\%) had pulmonary effusion caused by hypoproteinemia due to chronic kidney disease and inadequate hemodialysis. Secondly, some hemodialysis patients experienced extended periods of lung inflammation and pleural thickening due to long-term accumulation of fluid in the lungs. Almost all hemodialysis patients had anemia, causing an increased burden on the heart and shortness of breath. Hemodialysis patients also had a lower incidence of fever, which may be related to immunosuppression caused by uremia.

All hemodialysis patients presented on chest CT with typical signs of a viral infection, 
165 hemodialysis had pulmonary effusion due to inadequate hemodialysis and chronic lung 166 inflammation caused by uremia and pulmonary effusion, which may contribute to CT features such 167 as cord-like high-density shadows, pleural thickening, atelectasis, and pulmonary fibrosis. 168 Healthcare-associated pneumonia (HDAP) is commonly contracted by hemodialysis patients 169 (Carratalà \& Garcia-Vidal, 2008; Lee \& Moon, 2016). Therefore, these CT features may make the 170 diagnosis of COVID-19 infection more difficult in hemodialysis patients, compared with non171 dialysis patients.

172 The majority of the hemodialysis patients with COVID-19 in our study were middle-aged and 173 senile; 12 patients (75\%) had comorbidities, including coronary disease, hypertension, and 174 diabetes. Most hemodialysis patients were immunocompromised due to uremia (Betjes, 2013; Kim 175 et al., 2017; Syed-Ahmed \& Narayanan, 2019), which may have led to longer hospital stays and 176 higher mortality than for those in the general population.

177 Almost all hemodialysis patients had anemia, hypoproteinemia, and lymphopenia coagulopathy with elevated D-dimer at admission, increasing the risk of an unfavorable prognosis. This is keeping with the finding of a previous study that reported that severe lymphopenia and elevated

D-dimer were risk factors for the prognosis of patients with COVID-19 (Zhou et al., 2020).

However, in hemodialysis patients, elevated D-dimer may be partly caused by uremia and hemodialysis, therefore we could not simply consider D-dimer levels as risk factors for mortality in hemodialysis patients with COVID-19, as in the general population.

SARS-CoV-2, which is responsible for COVID-19, belongs to the $\beta$-type coronavirus, is 185 enveloped, has round or oval particles, and is often polymorphic, with a diameter of $60-140 \mathrm{~nm}$ 
186 (Shang et al., 2020). Current research has shown that SARS-CoV-2 has more than 79\% homology 187 with the severe acute respiratory syndrome (SARS) coronavirus, and the S proteins of SARS-CoV1882 and SARS-CoV, have a sequence similarity of approximately 77\% (Lu et al., 2020; Yuan et al., 189 2020). However, clinical studies have revealed many differences between SARS-CoV-2 and 190 SARS coronavirus. For example, SARS-CoV-2 has higher infectivity and lower lethality compared with SARS coronavirus (Guan et al., 2020; Park et al., 2020; Xu et al., 2020; Zhang et al., 2020). A study on severe acute respiratory syndrome reported that dialysis patients had similar clinical features and mortality rates as non-dialysis patients, but dialysis patients tended to present with less pronounced symptoms and had a much longer hospital stay compared with non-uremic patients (BC et al., 2004). The dialysis patients we studied tended to have a lower incidence of fever but a higher incidence of shortness of breath and unfavorable clinical prognosis, which may be due to the greater differences in the pathogenic mechanisms of the two viruses. Additional research is needed to validate these findings.

Our study was limited by its small sample size and single center demographics. A systematic and comprehensive study should be conducted to include a larger sample size across multiple centers to better assess the effects of COVID-19 infections in hemodialysis patients. In addition, we were only able to collect the information from hemodialysis patients with COVID-19, but we were not able to compare the clinical characteristics of hemodialysis patients with or without SARS-CoV2, nor can we describe the epidemiology of COVID-19 infections in hemodialysis patients. However, our study details fundamental information about the characteristics of COVID-19 in this patient population. 
207

208

209

210

211

212

213

214

215

216

217

218

219

220

221

222

223

224

225

226

227

228

229

230

231

232

233

234

In summary, we described the clinical characteristics and outcomes of hemodialysis patients with

COVID-19 infections. Hemodialysis patients are a high-risk group for infection by COVID-19.

They presented with similar clinical characteristics to those who were not on hemodialysis.

Hemodialysis patients had multiple comorbidities and worse physical conditioning, prolonged

hospital stays, and unfavorable clinical prognoses. Therefore, hemodialysis patients should be

monitored intensively for COVID-19 infections.

\section{Author contributions}

Dr. Luo and Cao had full access to all the data in the study and takes responsibility for the integrity of the data and the accuracy of the data analysis. Study concept and design: J.L. Li, C.A. Cao, Y.W. Luo. Acquisition, analysis, or interpretation of data: Y.W. Luo, Z.F. Liu, J.L. Li, X. Peng. Drafting of the manuscript: J.L. Li, Y.W. Luo. Critical revision of the manuscript for important intellectual content: C.A. Cao, X. Peng. Statistical analysis: J.L. Li, Y.W. Luo. Administrative, technical, or material support: J.L. Li, Z.F. Liu, X. Peng. Study supervision: C.A. Cao.

\section{Acknowledgments}

We thank the patients and their family members for their participation in our study.

\section{Reference}

WHO. Clinical management of severe acute respiratory infection when Novel coronavirus (nCoV) infection is suspected: interim guidance. Jan 11, 2020. https://www.who.int/docs/defaultsource/coronaviruse/clinical-management-of-novel-cov.pdf (accessed Apr. 5, 2020). 10.1097/01.asn.0000131522.16404.1f

CDC COVID-19 Response Team. Severe Outcomes Among Patients with Coronavirus Disease 2019 (COVID-19) - United States, February 12-March 16, 2020. MMWR Morb Mortal Wkly Rep. 2020 Mar 27;69(12):343-346. 10.15585/mmwr.mm6912e2. 
235

236

237

238

239

240

241

242

243

244

245

246

247

248

249

250

251

252

253

254

255

256

257

258

259

260

261

262

263

264

265

266

267

268

269

270

271

272

273

274

275

BC K, CB L, CC S, VW W, YL C, AW Y, and PK L. 2004. Severe acute respiratory syndrome in dialysis patients. J Am Soc Nephrol 15:1883-1888. 10.1097/01.asn.0000131522.16404.1f

Betjes MG. 2013. Immune cell dysfunction and inflammation in end-stage renal disease. Nat Rev Nephrol 9:255-265. 10.1038/nrneph.2013.44

Carratalà J, and Garcia-Vidal C. 2008. What is healthcare-associated pneumonia and how is it managed? Curr Opin Infect Dis 21:168-173. 10.1097/QCO.0b013e3282f4f248

CDC. 2020. Chinese diagnosis and treatment plan of COVID-19 patients (The sixth edition). http://www.nhc.gov.cn/yzygj/s7653p/202002/8334a8326dd94d329df351d7da8aefc2.shtm 1 .

Chen N, Zhou M, Dong X, Qu J, Gong F, Han Y, Qiu Y, Wang J, Liu Y, Wei Y, Xia J, Yu T, Zhang X, and Zhang L. 2020. Epidemiological and clinical characteristics of 99 cases of 2019 novel coronavirus pneumonia in Wuhan, China: a descriptive study. Lancet 395:507513. 10.1016/s0140-6736(20)30211-7

Cortes Garcia M, Sierra Moros MJ, Santa-Olalla Peralta P, Hernandez-Barrera V, Jimenez-Garcia R, and Pachon I. 2012. Clinical characteristics and outcomes of diabetic patients who were hospitalised with 2009 pandemic influenza A H1N1 infection. $J$ Infect 64:218-224. 10.1016/j.jinf.2011.11.022

Guan W-j, Ni Z-y, Hu Y, Liang W-h, Ou C-q, He J-x, Liu L, Shan H, Lei C-1, Hui DSC, Du B, Li L-j, Zeng G, Yuen K-Y, Chen R-c, Tang C-l, Wang T, Chen P-y, Xiang J, Li S-y, Wang J-1, Liang Z-j, Peng Y-x, Wei L, Liu Y, Hu Y-h, Peng P, Wang J-m, Liu J-y, Chen Z, Li G, Zheng Z-j, Qiu S-q, Luo J, Ye C-j, Zhu S-y, and Zhong N-s. 2020. Clinical Characteristics of Coronavirus Disease 2019 in China. N Engl J Med. 10.1056/NEJMoa2002032

Kim JU, Kim M, Kim S, Nguyen TT, Kim E, Lee S, Kim S, and Kim H. 2017. Dendritic Cell Dysfunction in Patients with End-stage Renal Disease. Immune Netw 17:152-162. 10.4110/in.2017.17.3.152

Lee JH, and Moon JC. 2016. Clinical characteristics of patients with hemodialysis-associated pneumonia compared to patients with non-hemodialysis community-onset pneumonia. Respir Med 111:84-90. 10.1016/j.rmed.2015.12.009

Lovshin JA, Bjornstad P, Lovblom LE, Bai JW, Lytvyn Y, Boulet G, Farooqi MA, Santiago S, Orszag A, Scarr D, Weisman A, Keenan HA, Brent MH, Paul N, Bril V, Perkins BA, and Cherney DZI. 2018. Atherosclerosis and Microvascular Complications: Results From the Canadian Study of Longevity in Type 1 Diabetes. Diabetes Care 41:2570-2578. 10.2337/dc18-1236

Lu R, Zhao X, Li J, Niu P, Yang B, Wu H, Wang W, Song H, Huang B, Zhu N, Bi Y, Ma X, Zhan F, Wang L, Hu T, Zhou H, Hu Z, Zhou W, Zhao L, Chen J, Meng Y, Wang J, Lin Y, Yuan J, Xie Z, Ma J, Liu WJ, Wang D, Xu W, Holmes EC, Gao GF, Wu G, Chen W, Shi W, and Tan W. 2020. Genomic characterisation and epidemiology of 2019 novel coronavirus: implications for virus origins and receptor binding. Lancet 395:565-574. 10.1016/s01406736(20)30251-8

Park M, Cook AR, Lim JT, Sun Y, and Dickens BL. 2020. A Systematic Review of COVID-19 Epidemiology Based on Current Evidence. J Clin Med 9:967. 10.3390/jcm9040967 
276

277

278

279

280

281

282

283

284

285

286

287

288

289

290

291

292

293

294

295

296

297

298

299

300

301

302

303

304

305

306

307

308

309

310

311

312

313

314

315

316

Shang J, Ye G, Shi K, Wan Y, Luo C, Aihara H, Geng Q, Auerbach A, and Li F. 2020. Structural basis of receptor recognition by SARS-CoV-2. Nature 581:221-224. 10.1038/s41586-0202179-y

Syed-Ahmed M, and Narayanan M. 2019. Immune Dysfunction and Risk of Infection in Chronic Kidney Disease. Adv Chronic Kidney Dis 26:8-15. 10.1053/j.ackd.2019.01.004

Tortonese S, Scriabine I, Anjou L, Loens C, Michon A, Benabdelhak M, Ouali S, Morin G, Laifi M, Dobosziewicz H, Guillet M, Dekeyser M, Luong Nguyen LB, Grünenwald A, Dang J, Desbuissons G, Becquemont L, Snanoudj R, Legendre C, Hebibi H, Lefèvre E, Beaudreuil S, and Zaidan M. 2020. COVID-19 in Patients on Maintenance Dialysis in the Paris Region. Kidney Int Rep. 10.1016/j.ekir.2020.07.016

Wang D, Hu B, Hu C, Zhu F, Liu X, Zhang J, Wang B, Xiang H, Cheng Z, Xiong Y, Zhao Y, Li Y, Wang X, and Peng Z. 2020a. Clinical Characteristics of 138 Hospitalized Patients With 2019 Novel Coronavirus-Infected Pneumonia in Wuhan, China. Jama 323:1061-1069. 10.1001/jama.2020.1585

Wang Y, Hu M, Ye G, Zhao Y, Yin Q, Pi P, and Luo D. 2020b. Clinical characteristics of patients with uremia undergoing maintenance hemodialysis complicated with COVID-19. Medicine (Baltimore) 99:e21547. 10.1097/md.0000000000021547

WHO. 2020. Coronavirus disease (COVID-2019) situation reports. Augest. 28, 2020. https://www.who.int/docs/default-source/coronaviruse/situation-reports/wou-28-augustapproved.pdf? sfvrsn=d9e49c20_2 (accessed Aug. 28, 2020).

Wu J, Li J, Zhu G, Zhang Y, Bi Z, Yu Y, Huang B, Fu S, Tan Y, Sun J, and Li X. 2020. Clinical Features of Maintenance Hemodialysis Patients with 2019 Novel Coronavirus-Infected Pneumonia in Wuhan, China. Clin $J$ Am Soc Nephrol 15:1139-1145. $10.2215 /$ cjn. 04160320

Wu Z, and McGoogan JM. 2020. Characteristics of and Important Lessons From the Coronavirus Disease 2019 (COVID-19) Outbreak in China: Summary of a Report of 72314 Cases From the Chinese Center for Disease Control and Prevention. Jama. 10.1001/jama.2020.2648

Xiong F, Tang H, Liu L, Tu C, Tian JB, Lei CT, Liu J, Dong JW, Chen WL, Wang XH, Luo D, Shi M, Miao XP, and Zhang C. 2020. Clinical Characteristics of and Medical Interventions for COVID-19 in Hemodialysis Patients in Wuhan, China. J Am Soc Nephrol 31:13871397. 10.1681/asn.2020030354

Xu X-W, Wu X-X, Jiang X-G, Xu K-J, Ying L-J, Ma C-L, Li S-B, Wang H-Y, Zhang S, Gao HN, Sheng J-F, Cai H-L, Qiu Y-Q, and Li L-J. 2020. Clinical findings in a group of patients infected with the 2019 novel coronavirus (SARS-Cov-2) outside of Wuhan, China: retrospective case series. BMJ 368:m606. 10.1136/bmj.m606

Yang D, Xiao Y, Chen J, Chen Y, Luo P, Liu Q, Yang C, Xiong M, Zhang Y, Liu X, Chen H, Deng A, Huang K, Cheng B, and Peng A. 2020. COVID-19 \& Chronic Renal Disease: Clinical characteristics \& prognosis. Qjm. 10.1093/qjmed/hcaa258

Yuan M, Wu NC, Zhu X, Lee CD, So RTY, Lv H, Mok CKP, and Wilson IA. 2020. A highly conserved cryptic epitope in the receptor binding domains of SARS-CoV-2 and SARSCoV. Science 368:630-633. 10.1126/science.abb7269 
317

318

319

320

321

322

323

324

325

326

327
Zhang J, Litvinova M, Wang W, Wang Y, Deng X, Chen X, Li M, Zheng W, Yi L, Chen X, Wu Q, Liang Y, Wang X, Yang J, Sun K, Longini IM, Jr., Halloran ME, Wu P, Cowling BJ, Merler S, Viboud C, Vespignani A, Ajelli M, and Yu H. 2020. Evolving epidemiology and transmission dynamics of coronavirus disease 2019 outside Hubei province, China: a descriptive and modelling study. Lancet Infect Dis Published online April 2, 2020. doi: 10.1016/S1473-3099(20)30230-9. 10.1016/s1473-3099(20)30230-9

Zhou F, Yu T, Du R, Fan G, Liu Y, Liu Z, Xiang J, Wang Y, Song B, Gu X, Guan L, Wei Y, Li $\mathrm{H}, \mathrm{Wu}$ X, Xu J, Tu S, Zhang Y, Chen H, and Cao B. 2020. Clinical course and risk factors for mortality of adult inpatients with COVID-19 in Wuhan, China: a retrospective cohort study. Lancet 395:1054-1062. 10.1016/s0140-6736(20)30566-3 


\section{Table $\mathbf{1}$ (on next page)}

Table 1

Clinical characteristics of hemodialysis patients with COVID-19 
Table 1 Clinical characteristics of hemodialysis and non-dialysis patients with COVID-19

\begin{tabular}{|c|c|c|c|}
\hline \multirow{2}{*}{ Characteristics } & \multicolumn{2}{|c|}{ No. (\%) } & \multirow{2}{*}{$P$ value } \\
\hline & Hemodialysis Patients & Non-dialysis Patients & \\
\hline Age (years), median (IQR) & $61(54-78)$ & $62(50-70)$ & 0.439 \\
\hline Male & $8(50)$ & $33(53.2)$ & 0.755 \\
\hline Contact history of epidemic area & $16(100)$ & $62(100)$ & $>0.99$ \\
\hline Preadmission comorbidities & $12(75)$ & $38(61)$ & 0.467 \\
\hline Hypertension & $11(68.75)$ & $19(30.65)$ & 0.012 \\
\hline Diabetes & $3(18.75)$ & $11(17.74)$ & $>0.99$ \\
\hline Cardiovascular diseases & $4(25)$ & $4(6.45)$ & 0.086 \\
\hline Cerebrovascular diseases & $0(0)$ & $2(3.23)$ & $>0.99$ \\
\hline Malignancy & $1(6.25)$ & $2(3.23)$ & $>0.99$ \\
\hline COPD & $0(0)$ & $2(6.45)$ & $>0.99$ \\
\hline Tuberculosis & $0(0)$ & $2(3.23)$ & $>0.99$ \\
\hline \multicolumn{4}{|l|}{ Pneumonia related manifestations } \\
\hline Cough & $12(75)$ & $45(72.58)$ & 0.699 \\
\hline shortness of breath & $8(50)$ & $19(33.87)$ & 0.248 \\
\hline Fatigue & $8(50)$ & $42(67.74)$ & 0.305 \\
\hline Fever & $7(43.75)$ & $54(87.10)$ & 0.001 \\
\hline Diarrhoea & $2(12.5)$ & $11(17.74)$ & 0.900 \\
\hline Myalgia & $2(12.5)$ & $23(37.10)$ & 0.275 \\
\hline Headache & $1(6.25)$ & $2(3.22)$ & $>0.99$ \\
\hline Sore throat & $1(6.25)$ & $3(4.84)$ & $>0.99$ \\
\hline Incubation period (days), median (IQR) & $9(4.3-12)$ & $8(5-12)$ & 0.674 \\
\hline
\end{tabular}

3 Data are reported as n (\%) or median (IQR). The $P$ value represents the difference between hemodialysis and non-dialysis patients. $P$ value $<0.05$ was

4 considered significant difference (Bold font) . 


\section{Table 2 (on next page)}

Table 2

Laboratory results of Hemodialysis patients with COVID-19 
Table 2 Laboratory results of hemodialysis and non-dialysis patients with COVID-19

\begin{tabular}{|c|c|c|c|}
\hline \multirow{2}{*}{ Characteristics } & \multicolumn{2}{|c|}{ No. $(\%)$} & \multirow{2}{*}{$P$ value } \\
\hline & Hemodialysis Patients & Non-dialysis Patients & \\
\hline \multicolumn{4}{|c|}{ Admission radiologic findings (Chest CT) } \\
\hline Bilateral patchy shadowing & $16(100)$ & $58(93.5)$ & 0.683 \\
\hline Ground-glass opacities & $10(62.5)$ & $47(75.8)$ & 0.451 \\
\hline Pulmonary effusion & $8(50)$ & $2(3.23)$ & $<0.001$ \\
\hline Cord high density shadows & $7(43.75)$ & $4(6.45)$ & $<0.001$ \\
\hline Pleural thickening & $4(25)$ & $1(1.6)$ & 0.0046 \\
\hline Atelectasis & $4(25)$ & $1(1.6)$ & 0.0046 \\
\hline Consolidation of lung & $2(12.5)$ & $0(0)$ & 0.053 \\
\hline \multicolumn{4}{|l|}{ Blood routine } \\
\hline Hemoglobin $(\mathrm{g} / \mathrm{L})$ & $91.5(79.25-102.5)$ & $127.5(115-141.75)$ & $<0.001$ \\
\hline White blood cell count $\left(\times 10^{\wedge} 9 / \mathrm{L}\right)$ & $4.29(3.56-6.69)$ & $6.22(4.66-8.38)$ & 0.015 \\
\hline Neutrophil $\left(\times 10^{\wedge} 9 / \mathrm{L}\right)$ & $3.19(2.47-5.3)$ & $5.18(3.27-7.44)$ & 0.016 \\
\hline Lymphocyte count $\left(\times 10^{\wedge} 9 / \mathrm{L}\right)$ & $0.65(0.51-0.91)$ & $0.74(0.40-1.17)$ & 0.656 \\
\hline \multicolumn{4}{|l|}{ Coagulation function } \\
\hline APTT & $31.5(26.6-43.3)$ & $35.5(32.13-38.32)$ & 0.290 \\
\hline Prothrombin time (s) & $12.2(11.05-14.13)$ & $12.9(12.3-13.33)$ & 0.133 \\
\hline $\mathrm{TT}$ & $19.5(18.9-20.98)$ & $15.7(14.7-16.5)$ & $<0.001$ \\
\hline $\mathrm{D}$-dimer $(\mathrm{mg} / \mathrm{L})$ & $1.84(0.88-4.55)$ & $0.49(0.24-1.27)$ & $<0.001$ \\
\hline \multicolumn{4}{|l|}{ Blood biochemistry } \\
\hline AST (U/L) & $20(13-24)$ & $25.5(17-42.25)$ & $\mathbf{0 . 0 3 7}$ \\
\hline ALT (U/L) & $10(6.25-13.5)$ & $28(16-42.25)$ & $<0.001$ \\
\hline Serum creatinine $(\mu \mathrm{mol} / \mathrm{L})$ & $1067.5(855.08-1392.23)$ & $70.5(55.75-82.25)$ & $<0.001$ \\
\hline \multicolumn{4}{|l|}{ Infection-related biomarkers } \\
\hline C-reactive protein $(\mathrm{mg} / \mathrm{L})$ & $39.3(23.85-87.53)$ & $44.74(20.73-74.20)$ & 0.782 \\
\hline Procalcitonin $(\mathrm{ng} / \mathrm{mL})$ & $0.85(0.44-2.79)$ & $0.04(0.04-0.06)$ & $<0.001$ \\
\hline
\end{tabular}


Data are reported as n (\%) or median (IQR). The $P$ value represents the difference between hemodialysis and non-dialysis patients. $P$ value $<0.05$ was considered significant difference (Bold font) . 
Figure 1

Figure 1

Chest CT imaging of four hemodialysis patients
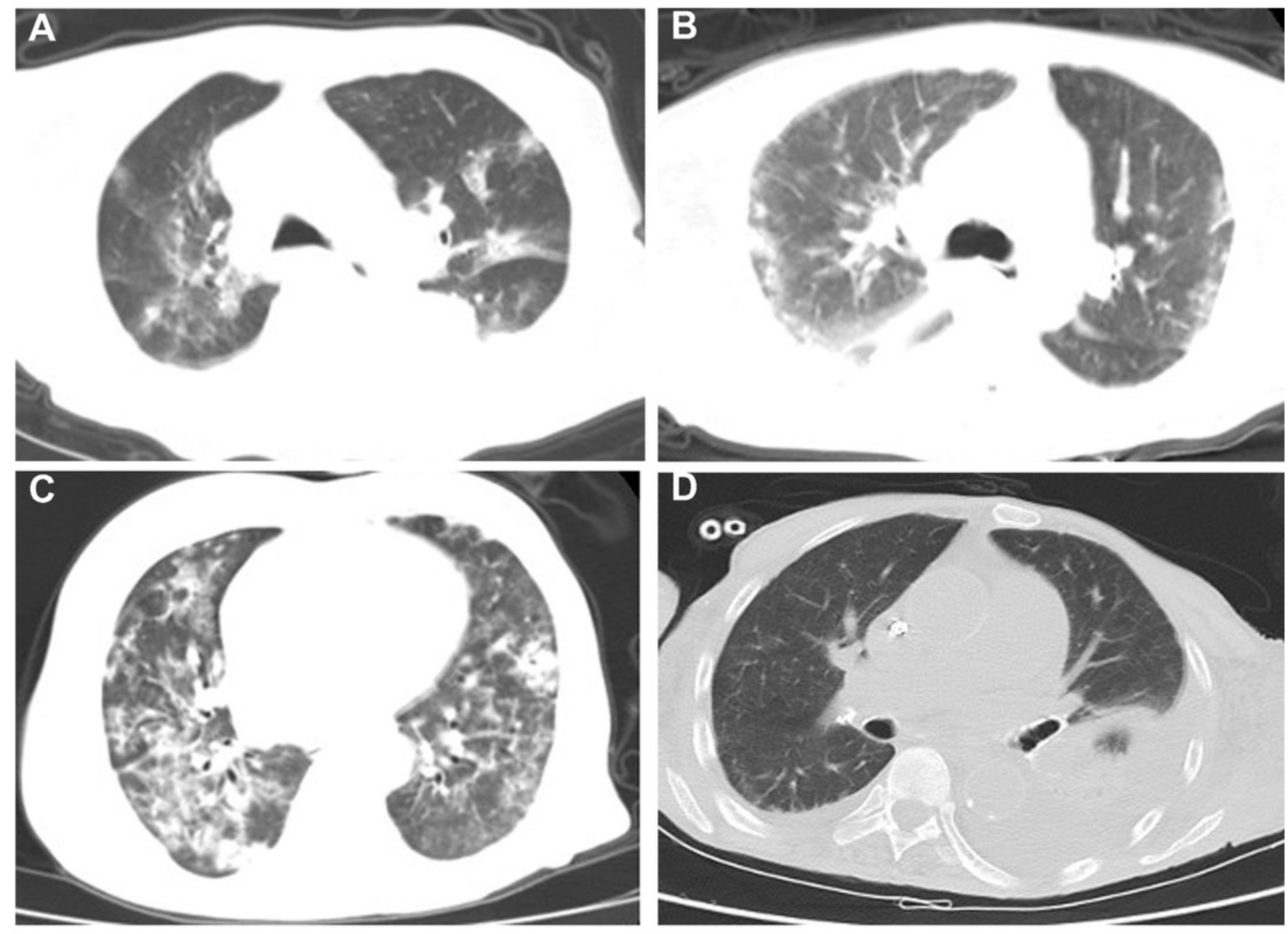